\title{
An immunological method to screen sex-specific proteins of bovine sperm
}

\author{
L. Sang, ${ }^{\star}$ W. C. Yang, ${ }^{\star}$ L. Han,† A. X. Liang, ${ }^{\star}$ G. H. Hua, ${ }^{\star}$ J. J. Xiong, ${ }^{\star}$ L. J. Huo, ${ }^{*}$ and L. G. Yang ${ }^{\star 1}$ \\ *Key Laboratory for Agricultural Animal Genetics, Breeding, and Reproduction, under Education Ministry of China, \\ College of Animal Science and Technology, Huazhong Agricultural University, Wuhan 430070, P.R. China \\ †State Key Laboratory of Agricultural Microbiology, College of Veterinary Medicine, Huazhong Agricultural University, Wuhan 430070, P.R. China
}

\begin{abstract}
This study was designed to identify sex-specific antibodies (SSAb) in rabbit antisera against bovine sex-sorted sperm, and capture sex-specific proteins of bovine X- or Y- proteins by SSAb. The rabbit antisera against bovine $\mathrm{X}$ - or $\mathrm{Y}$-sperm were first produced by a series of immunological approaches, and further purified through immuno-neutralization with excess sex-sorted Y- or X-sperm, respectively, to remove non-sex specific antibodies and enrich sex-specific antibodies. After removal of non-sex specific antibodies, the purified rabbit sera with enriched sex-specific antibodies were screened for sex-specific antibodies by immunofluorescence staining and flow cytometry. The results showed that 3.0, 2.2 , and $4.2 \%$ of unsorted sperm, sex-sorted X-sperm, and sex-sorted $Y$-sperm were recognized by the purified rabbit antisera against Y-sperm, respectively, whereas $29.2,19.7$, and $3.9 \%$ of unsorted sperm, sex-sorted Xsperm, and sex-sorted Y-sperm were recognized by the purified rabbit antisera against $\mathrm{X}$-sperm. These results suggested that the purified rabbit antisera against Xsperm contained SSAb that preferentially bound to sex-sorted X-sperm. Subsequently, the purified rabbit antisera against X- or Y-sperm were used to immunoprecipitate sex-specific proteins in bovine sperm proteins, and a $30-\mathrm{kDa}$ protein was specifically captured by the rabbit antisera against X-sperm. In conclusion, our results implied that this $30-\mathrm{kDa}$ protein might be a sex-specific protein in bovine X-sperm, which has the potential to be used in immunological procedures for sexing sperm.
\end{abstract}

Key words: sperm, sex-specific protein, flow cytometry, bovine

\section{INTRODUCTION}

The technology of controlling the sex of mammalian offspring is of great importance in both the livestock industry and human medicine (Garner and Seidel, 2008; Karabinus, 2009). An obvious way to achieve this

Received April 19, 2010.

Accepted December 14, 2010.

${ }^{1}$ Corresponding author: yangliguo2006@yahoo.com.cn is to sort $\mathrm{X}$ - and Y-sperm before conception, because it provides economically flexible management practices for animal producers (Rath et al., 2009). Of the potentially available methods, only flow cytometry can sort sperm at greater than $90 \%$ accuracy, resulting in offspring whose phenotypic sex is consistent with initial purity of the sex-sorted sperm population (Seidel, 2009). This technology has been applied in sorting sperm in many species of animals (O'Brien et al., 2009), and has reached a development level that allows its commercial application (Rath et al., 2009).

Although clearly effective, the use of flow cytometry as a routine tool for sex selection in animal breeding is relatively inefficient, requires specialized, expensive, and immobile equipment and highly skilled operators (Hendriksen, 1999). Furthermore, the sorting procedure likely causes damage to sperm (Spinaci et al., 2006; Bermejo-Álvarez et al., 2008). However, the use of flow cytometry also provides a new opportunity to discover differences in proteins between separated populations of $\mathrm{X}$ - and Y-sperm, which might be used in designing methods to produce large numbers of separated sperm. In this respect, the specific surface components of separated sperm are particularly interesting, because differences in membrane antigenicity could be exploited to generate antibodies capable of separating X- or Ysperm. Therefore, many attempts to search for differences between $\mathrm{X}$ - and Y-sperm have been described using 2-dimensional electrophoresis procedures, but available information about this technique failed to provide any evidence of the sex-specific proteins (SSP; Hendriksen et al., 1996; Hendriksen, 1999). However, much indirect evidence implied that differences might exist between X- and Y-sperm (Grant et al., 2008; Robbins et al., 2008; Zhang et al., 2008). Due to lack of success, it is speculated that differences in protein composition between X- and Y-sperm might occur for a minor component of the membrane with a low antigenicity or carbohydrate epitope (Soares and Barbosa, 2008), and membrane proteins might be below the present detection level by 2-dimensional gel electrophoresis (Hendriksen et al., 1996).

In principle, each cellular protein is recognizable by a set of antibodies, so an immunological approach may 
offer an alternative and perhaps more efficient way for searching SSP (Beerli et al., 2008). A series of immunological approaches have been used to identify sperm antigens binding to the zona pellucida (Sivapurapu et al., 2003), and to isolate capacitation-related antigens (Mollova et al., 1999) and other antigens with interesting functions (Khan et al., 2009). Thus, Blecher et al. (1999) attempted to isolate sex-specific proteins by male rabbit sera against unsorted sperm and female rabbit sera against unsorted sperm, and the female rabbit sera against unsorted sperm could cause agglutination of approximately half of the bovine sperm and when the unagglutinated sperm were isolated and used in bovine in vitro fertilization, they produced above $90 \%$ male embryos. However, to date, producing sex-selected offspring with this procedure has not been reported, despite considerable investment of resources over several years. Simultaneously, Soares and Barbosa (2008) also have used an antibody phage display technique to identify sex-specific antibodies (SSAb) in an antibody recombinant library, and a positive clone was found to preferentially bind to male leukocytes. Although the direct evidence of sex-specific proteins is still unclear, it was feasible that immunological methods could be applied to search for sex-specific antigens that are expressed at relatively low levels.

In this study, rabbit antisera against sex-sorted bovine $\mathrm{X}$ - or $\mathrm{Y}$-sperm were produced, and further purified by immuno-neutralization with excess sex-sorted Y- or $\mathrm{X}$-sperm, respectively, to remove non-sex-specific antibodies (non-SSAb). It was investigated whether SSAb were present in the rabbit antisera against bovine Xor Y-sperm by a series of immunological approaches, and whether SSP were captured by the purified rabbit antisera against sex-sorted sperm. It might provide some assistance to develop an efficient, inexpensive, convenient technology for sorting sperm, and investigate a molecular mechanism for controlling the sex of mammals.

\section{MATERIALS AND METHODS}

\section{Preparation of Bovine Sperm Proteins}

The extracted proteins from sperm were prepared, and the methods used were described in a publication by Harlow and Lane (1999). Semen from Holstein bulls of proven fertility (provided by Breeding Bull Station of Hubei Province, China) was washed with an equal volume of a cushion of $0.25 \mathrm{M}$ sucrose in $10 \mathrm{mM}$ Tris$\mathrm{HCl}, \mathrm{pH}$ 7.4. The sample was centrifuged at $450 \times g$ for $10 \mathrm{~min}$ at room temperature (approximately $20^{\circ} \mathrm{C}$ ), and the supernatant was aspirated to leave a loose pellet of sperm. The sperm pellet was resuspended in $9 \mathrm{~mL}$ of
$0.01 M$ PBS $(\mathrm{pH} 7.4$ ), and centrifuged at $450 \times g$ for $10 \mathrm{~min}$ at room temperature (Howes et al., 1997). This procedure was repeated twice. One hundred microliters of denaturing lysis buffer containing $20 \mathrm{~m} M$ Tris- $\mathrm{HCl}$ (pH 7.4), $150 \mathrm{mM} \mathrm{NaCl}, 1 \%$ SDS, and 1\% Triton X-100 were added per $2 \times 10^{7}$ sperm in a pellet. The suspension was incubated at $95^{\circ} \mathrm{C}$ for 5 min and then cooled on ice for $5 \mathrm{~min}$. Afterward, the suspension was mixed with 9 volumes of nondenaturing lysis buffer containing $20 \mathrm{~m} M$ Tris- $\mathrm{HCl}(\mathrm{pH} \mathrm{7.4)}$ and $150 \mathrm{mM} \mathrm{NaCl}, 1 \%$ Triton X-100, and $1 \mathrm{~m} M$ phenylmethyl sulfonylfluoride (PMSF). Ten microliters of protease inhibitor cocktail (1:100, Roche, Mannheim, Germany) was added per 1 $\mathrm{mL}$ of the suspension. The sperm DNA was sheared by passing the suspension 5 to 10 times through a $25-\mathrm{G}$ needle attached to $1-\mathrm{mL}$ syringe. The suspension was centrifuged at $12,000 \times g$ for $10 \mathrm{~min}$ at $4^{\circ} \mathrm{C}$, and the supernatant was transferred to a fresh tube for immunoprecipitation. The protein concentration was determined by BCA Protein Assay Kit (Pierce, Rockford, IL).

\section{Production of Rabbit Antisera Against Sex-Sorted Sperm}

Animal care and handing were in accordance with the policy on the Care and Use of Animals of the Ethical Committee, Huazhong Agricultural University. Male Japanese white rabbits (3.0 to $3.5 \mathrm{~kg}$ and more than 6 mo old), purchased from the Medical Laboratory Animal Center of Hubei Province (Wuhan, China), were allowed free access to food and water at all times, and maintained on a $12 \mathrm{~h} \mathrm{~L} / 12 \mathrm{~h} \mathrm{D}$ (light/dark) cycle and in an air-conditioned room. Sex-sorted X- or Y-sperm from 3 Holstein bulls were purchased from XY Breeding Livestock Company (Tianjin, China). The result of reanalysis of sex-sorted sperm for sorting accuracy by a modified flow cytometer (MoFlo SX; Dako Colorado Inc., Fort Collins, $\mathrm{CO}$ ) showed that the efficiencies of flow cytometric sorting of either X-sperm $(\mathrm{n}=3)$ or Y-sperm $(\mathrm{n}=3)$ were all above $90 \%$ (provided by XY Breeding Livestock Company; Figure 1).

Male rabbits were immunized with pooled, sex-sorted $\mathrm{X}$ - or Y-sperm. Briefly, the sex-sorted X- or Y-sperm were washed twice in a cushion of $0.25 \mathrm{M}$ sucrose in 10 $\mathrm{m} M$ Tris- $\mathrm{HCl}, \mathrm{pH} 7.4$, with centrifugation at $200 \times g$ for $10 \mathrm{~min}$ at room temperature (Howes et al., 1997). Sperm counts were done using a hemacytometer. The rabbits were injected with the sex-sorted X- or Y-sperm at a dosage of $1 \times 10^{6}$ sperm $/ 125 \mu \mathrm{L}$, mixed with the same volume of Freund's complete adjuvant (Sigma Aldrich, Steinheim am Albuch, Germany), and preimmune sera were collected before immunization. After primary immunization, 3 booster immunizations were given at 
(a) Efficiencies of flow cytometric sorting of Y sperm
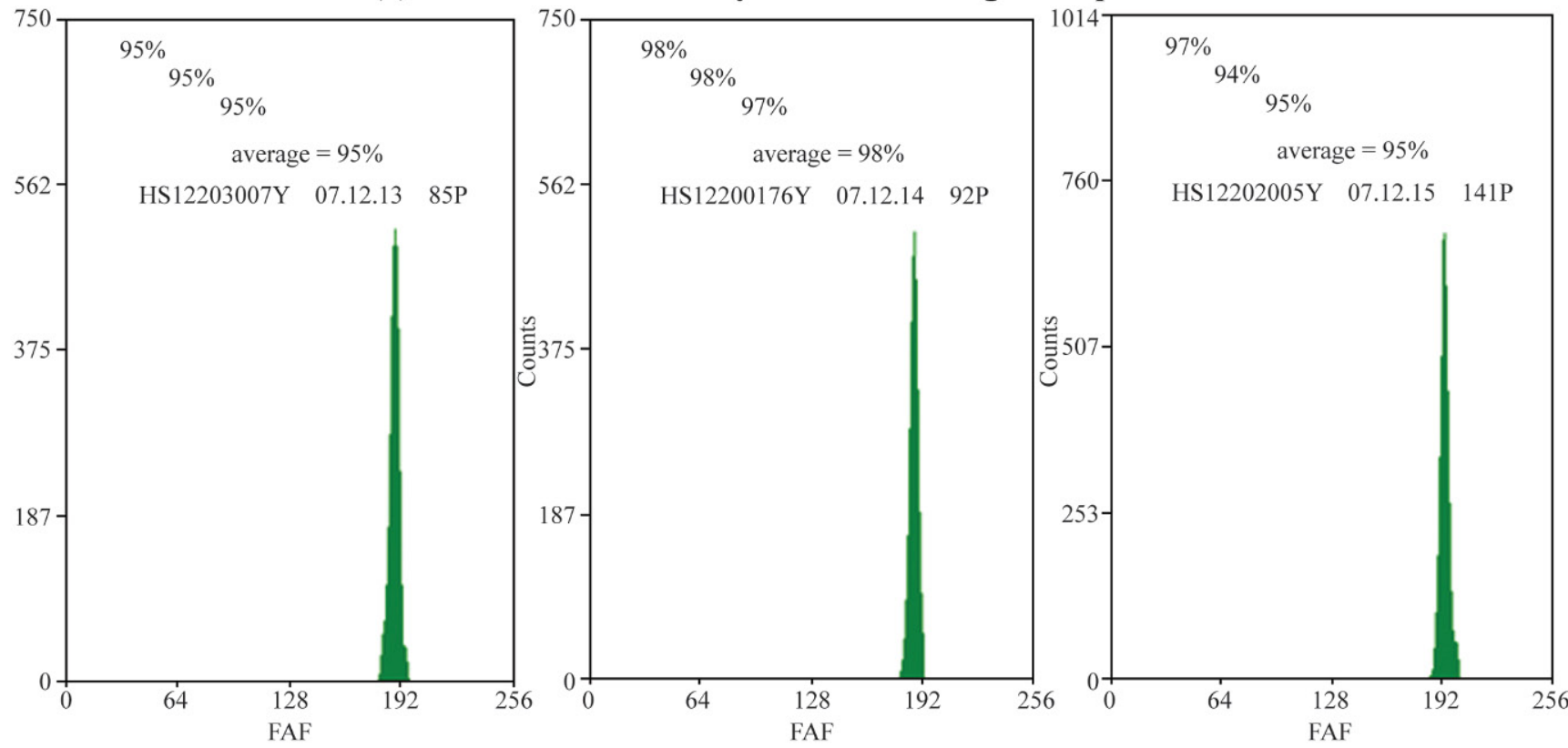

(b) Efficiencies of flow cytometric sorting of $\mathrm{X}$ sperm
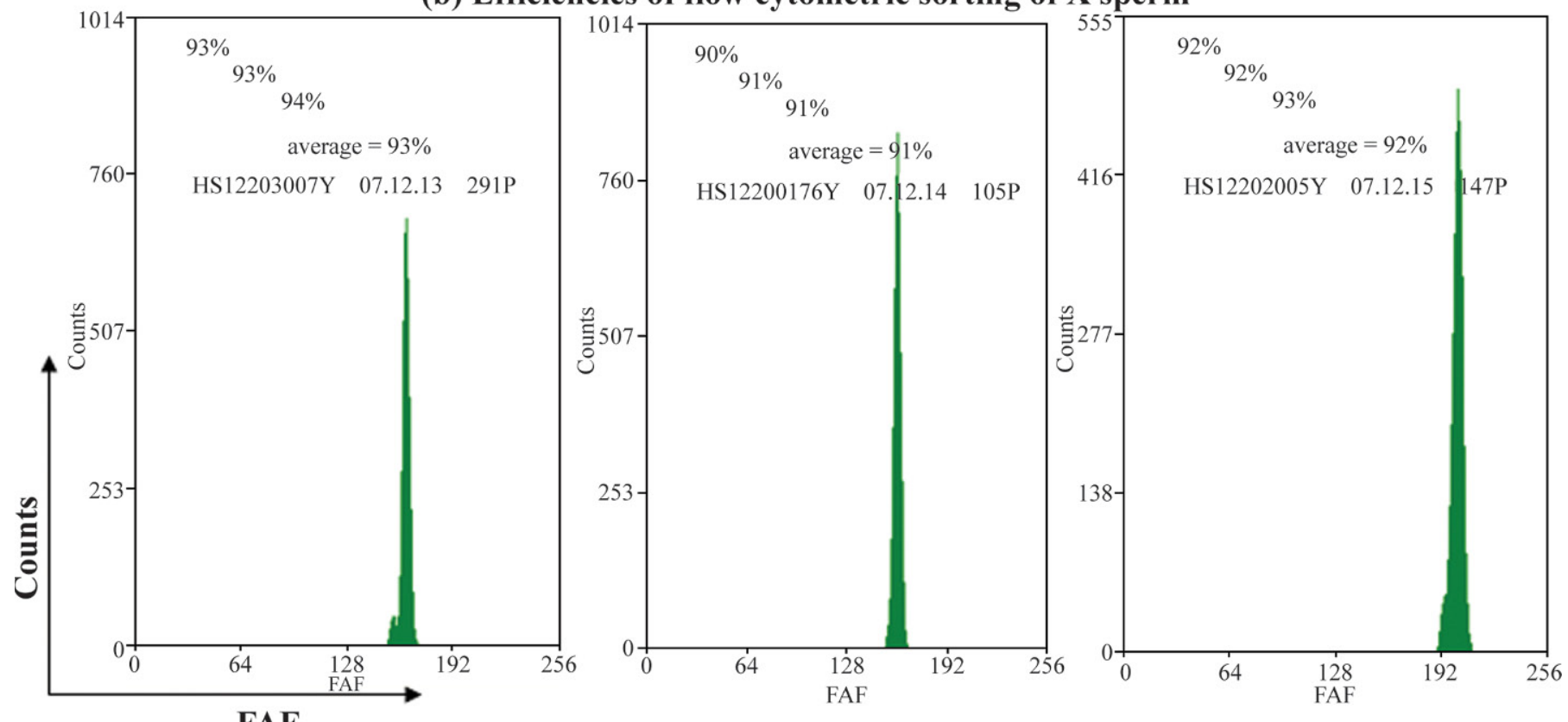

FAF

Figure 1. The result of reanalysis of sex-sorted sperm for sorting accuracy. Suitable aliquots of sex-sorted sperm from 3 Holstein bulls for sort reanalysis were sonicated to remove sperm tails, incubated with additional Hoechst 33342, and sex-sorted by a modified flow cytometer (MoFlo SX; Dako Colorado Inc., Fort Collins, CO). Approximately 5,000 fluorescent signals of bull sperm per sample were recorded in the reanalysis. Bull sperm sonicated to remove the tails and stained with Hoechst 33342 at the same concentration level were used for viable sperm preparations. The histogram represents the DNA content distribution of the properly oriented either X- or Y-sperm reanalyzed with forward angle fluorescence detector (FAF). Flow cytometric histogram outputs showed that the efficiencies of flow cytometric sorting of either X-sperm $(\mathrm{n}=3)$ or $\mathrm{Y}$-sperm $(\mathrm{n}=3)$ were all above $90 \%$. (Provided by XY Breeding Livestock Company, Tianjin, China). Color version available in the online PDF.

intervals of 1 wk using sex-sorted X- or Y-sperm at a dosage of $2 \times 10^{6}$ sperm $/ 250 \mu \mathrm{L}$ with the same volume of Freund's incomplete adjuvant (Sigma Aldrich) for the first 2 immunizations, and at a dosage of $4 \times 10^{6}$ sperm $/ 500 \mu \mathrm{L}$ without adjuvant for the third booster immunization. The blood samples were harvested by ear vein puncture with heparinized tubes on d 10 after the last immunization. Following centrifugation at 4,000 $\times$ 
$g$ for $5 \mathrm{~min}$, the sera were stored at $-20^{\circ} \mathrm{C}$ (Liang et al., 2008). Antibodies against sperm were evaluated with a cell-based ELISA method with minor modification (Rajeev and Reddy, 2004), using unsorted sperm as antigens. All sera from the experimental groups showed a strong immune response, whereas the preimmune sera were negative (data not shown).

\section{Screening of Antibodies Against X- or Y-Sperm by Immuno-Neutralization}

Screening of antibodies against X- or Y-sperm was performed via removal of non-SSAb in rabbit sera by immuno-neutralization. Before removal of the crossreactive antibodies against $\mathrm{X}$ - or $\mathrm{Y}$-sperm in the rabbit antisera, a preliminary experiment was used to determine the optimal condition of immuno-neutralization (Wang et al., 2003). The rabbit antisera against X- or Y-sperm were serially diluted ranging from 1:10 to $1: 1,280$ in PBS containing $0.1 \%$ Tween-20 and $1 \%$ bovine serum albumin (Roche Molecular Biochemicals, Mannheim, Germany). Different dilutions of the rabbit antisera against X- or Y-sperm were incubated with an equal volume of unsorted sperm at a concentration of $10^{8} / \mathrm{mL}$ for $2 \mathrm{~h}$ at $4^{\circ} \mathrm{C}$. Following centrifugation at $200 \times g$ for $10 \mathrm{~min}$, the supernatant were collected, and then evaluated for antibodies with a cell-based ELISA with minor modification (Rajeev and Reddy, 2004). Briefly, unsorted sperm at a concentration of $1 \times 10^{6} / \mathrm{mL}$ were coated onto 96-well immunoplates (Greiner bio-one, Frickenhausen, Germany) and incubated overnight at $4^{\circ} \mathrm{C}$. The sperm were fixed using $70 \%$ ethanol. The nonspecific sites were blocked with normal goat serum (Boster, Wuhan, China) at a dilution of 1:1,000. Endogenous peroxidase was quenched with $0.5 \% \mathrm{H}_{2} \mathrm{O}_{2}$ in PBS. The previously collected supernatant were added into wells and incubated at $37^{\circ} \mathrm{C}$ for $1 \mathrm{~h}$, followed by horseradish peroxidase conjugate goat anti-rabbit IgG (Boster) at a dilution of 1:2,500. In between each step, the plates were washed with PBS with $0.01 \%$ Tween-20 (PBST). To develop the ELISA result, $10 \mathrm{mg}$ of tetramethylbenzidine (TMB tablets, Sigma-Aldrich Chemical Co., St. Louis, MO), dissolved in absolute ethanol, was mixed with phosphate-citrate buffer ( $\mathrm{pH}$ 5.0) using $\mathrm{H}_{2} \mathrm{O}_{2}$ as the substrate, then incubated for $10 \mathrm{~min}$ at $37^{\circ} \mathrm{C}$. Reactions were terminated with $2 \mathrm{M} \mathrm{H}_{2} \mathrm{SO}_{4}$ and the resulting optical density (OD) was measured at $450 \mathrm{~nm}$ in a plate reader (Multiskan MK3, Thermo Labsystems, Franklin, MA). The optimal dilution titer of antisera was designed so that no antibodies were detected in the supernatant.

The optimally diluted antisera against X- or Y-sperm were incubated with an equal volume of sex-sorted Yor X-sperm at a concentration of $1 \times 10^{8} / \mathrm{mL}$ for $2 \mathrm{~h}$ at $4^{\circ} \mathrm{C}$, respectively. Following centrifugation at $200 \times$ $g$ for $10 \mathrm{~min}$, the supernatants were collected, and used as putative SSAb against either X-sperm (XSSAb) or Y-sperm (YSSAb).

\section{Screening of SSAb by Flow Cytometry Using Unsorted Sperm}

Holstein semen with motility over $80 \%$ were provided by Breeding Bull Station of Hubei Province (Wuhan, China), and successively washed in a sucrose cushion and PBS. Following fixing in $70 \%$ ethanol over $1 \mathrm{~h}$ at $-20^{\circ} \mathrm{C}$, the samples were washed in PBS, and centrifuged at $450 \times g$ for $10 \mathrm{~min}$. Then, the supernatant was aspirated to leave a pellet of unsorted sperm. Fifty microliters of the putative XSSAb or YSSAb were added to approximately $2 \times 10^{6}$ unsorted sperm that had been fixed previously. The mixture was incubated for $1 \mathrm{~h}$ at $37^{\circ} \mathrm{C}$ and washed twice in PBS. Bound antibodies were detected by the addition of fluorescein isothiocyanate (FITC)-conjugated goat anti-rabbit polyclonal antibodies (Boster) diluted 1/50 in PBST and incubated for $1 \mathrm{~h}$ at $37^{\circ} \mathrm{C}$. Following 3 washes in PBS, the sperm were analyzed in the presence of $10 \mu \mathrm{g} / \mathrm{mL}$ propidium iodide (PI; Sigma Aldrich, Steinheim am Albuch, Germany) to exclude fragments of sperm and other particles. The unneutralized antisera against sex-sorted X- or Y-sperm were used as a positive control, and the preimmune sera were used as negative controls. All samples were evaluated by flow cytometry using a Beckman Coulter EPICS XL (Beckman Coulter, Inc., Miami, FL), and data were analyzed by the EXPO32 ADC XL 4COLOR software program (Beckman Coulter, Inc.).

\section{Screening of SSAb by Flow Cytometry Using Sex-Sorted X- or Y-Sperm}

The washed sex-sorted X- or Y-sperm $\left(2 \times 10^{6}\right)$ were fixed in $70 \%$ ethanol over $1 \mathrm{~h}$ at $-20^{\circ} \mathrm{C}$ and washed twice in PBS. Fifty microliters of putative XSSAb or YSSAb were added to the fixed X- or Y-sperm, respectively, and the preimmune sera were used as negative controls. The same protocol for unsorted sperm was used for sex-sorted X- or Y-sperm.

\section{Identification of Sex-Specific Proteins}

An immunoprecipitation method was performed to detect sex-specific proteins (SSP) in X- or Y-sperm. Briefly, protein A agarose beads (Upstate, Temecula, CA) were prepared as described in the manufacturer's manual and were first incubated (pre-clearing) with sperm proteins. This was necessary for blocking nonspecific binding sites on agarose beads. Then, $50 \mu \mathrm{L}$ of 
putative XSSAb or YSSAb were incubated with $1 \mathrm{mg}$ of sperm proteins, respectively. The reaction mixture was gently rocked at $4^{\circ} \mathrm{C}$ overnight. The immunocomplex was captured by adding $100 \mu \mathrm{L}$ of washed protein A agarose bead slurry ( $50 \mu \mathrm{L}$ of packed beads), and the reaction mixture was gently rocked at $4^{\circ} \mathrm{C}$ for 2 h. After incubation, samples were pulled down $[5 \mathrm{~s}$ in the microcentrifuge (MIKRO 20, Hettich Zentrifugen, Tuttlingen, Germany) at 14,000 $\times \mathrm{g}$ ] and supernatants removed by gentle aspiration. The pellets were washed 3 times with ice-cold PBS. Final pellets were resuspended in $60 \mu \mathrm{L}$ of $2 \times$ Laemmli sample buffer and boiled for 5 min. Beads were collected by a microcentrifuge pulse. Supernatants were subjected to $10 \%$ SDS-PAGE, and gels were subjected to silver staining (Sambrook and Russell, 2001).

\section{Statistical Analysis}

The serially diluted antisera ranging from 1:10 to 1:1,280 were incubated with an equal volume of $1 \times$ $10^{8}$ unsorted sperm. The comparisons of mean OD values were performed between the neutralized antisera against sex-sorted sperm and the preimmune sera by the ANOVA program of the SPSS procedures (version 11.5; SPSS Inc., Chicago, IL).

\section{RESULTS}

\section{Identification and Purification of Rabbit Antisera Against X- or Y-Sperm}

All rabbit antisera from the experimental groups showed a strong immune response, and the cross-reactive antibodies against X- or Y-sperm were neutralized by excess volume of sex-sorted Y- or X-sperm, respectively. Then SSAb in the purified rabbit antisera against sexsorted X- or Y-sperm could be enriched. Previously, a cell-based ELISA method was applied to determine the optimal condition of the neutralization reaction of the antisera against sex-sorted sperm with excess unsorted sperm. When rabbit antisera were diluted $\geq 1: 40$, no significant difference in mean OD values between the preimmune sera and the neutralized rabbit antisera against sex-sorted $\mathrm{X}$-sperm was found $(P>0.05$; Figure 2 ) or between the preimmune sera and the neutralized antisera against sex-sorted $\mathrm{Y}$-sperm $(P>0.05$; Figure $3)$. Thus, rabbit antisera against $\mathrm{X}$ - or $\mathrm{Y}$-sperm were diluted at 1:40 in PBST, and neutralized by an equal volume of $1 \times 10^{8} / \mathrm{mL}$ either Y-sperm or X-sperm to prepare the putative XSSAb or YSSAb.

\section{The Identification of SSAb by Flow Cytometry Using Unsorted Sperm}

The putative XSSAb or YSSAb were added to about $2 \times 10^{6}$ washed unsorted sperm and bound antibod- ies were detected by FITC-conjugated goat anti-rabbit polyclonal antibodies using a Beckman Coulter EPICS XL equipped with a 15-milliwatt argon ion laser (Beckman Coulter; excitation wavelength: $488 \mathrm{~nm}$; FITC fluorescence emission: $525 \mathrm{~nm}$; PI fluorescence emission: $620 \mathrm{~nm})$. The sperm stained with both PI and FITCconjugated goat anti-rabbit polyclonal antibodies were evaluated to measure the population of sperm labeled with SSAb; $1 \times 10^{4}$ events were examined. In Figure 4 , the results showed that the putative XSSAb bound $29.2 \%$ of unsorted bovine sperm, and the putative YS$\mathrm{SAb}$ bound $3.0 \%$ of unsorted bovine sperm. On the assumption that equal numbers of $\mathrm{X}$ - and $\mathrm{Y}$-sperm are present in semen, no SSAb were present in the putative YSSAb or XSSAb, because only $62.7 \%$ and $77.2 \%$ of the unsorted bovine sperm were recognized by unneutralized antisera against X- and Y-sperm. Compared with the results obtained with the unneutralized antisera, the sperm labeled by the putative XSSAb accounted for $46.6 \%$ of total sperm recognized by the unneutralized antisera against X-sperm (29.2\%: $62.7 \%)$, but the sperm labeled by the putative YSSAb was only $3.9 \%$ of total sperm recognized by the unneutralized antisera against Y-sperm (3.0\%: 77.2\%; Table 1). These results indicated that only the putative XSSAb bound to approximately half of the unsorted sperm.

\section{The Identification of SSAb by Flow Cytometry Using Sex-Sorted X- or Y-Sperm}

The XSSAb or YSSAb were also added to about $2 \times$ $10^{6}$ sex-sorted X- or Y-sperm respectively, and bound antibodies were detected by FITC-conjugated goat anti-rabbit polyclonal antibodies using flow cytometry. It was found that $19.7 \%$ of sex-sorted X-sperm and 3.9\% of sex-sorted Y-sperm were recognized by the putative XSSAb, whereas only $2.2 \%$ of sex-sorted X-sperm and $4.2 \%$ of sex-sorted Y-sperm were recognized by the putative YSSAb (Figure 5).

\section{Capture of SSP by Immunoprecipitation Using Sex-Sorted X-or Y-Sperm}

Based on the results obtained above, we predicted that the putative XSSAb contained SSAb. To capture the corresponding SSP in X-sperm, the putative $\mathrm{XSSAb}$ or YSSAb were used to immunoprecipitate SSP in whole sperm proteins. The immunocomplexes were subjected to SDS-PAGE, and analysis of fractions revealed that a specific band of approximately $30 \mathrm{kDa}$ after staining with silver nitrate was precipitated by the putative XSSAb, and was not able to be detected by the putative YSSAb (Figure 6). 


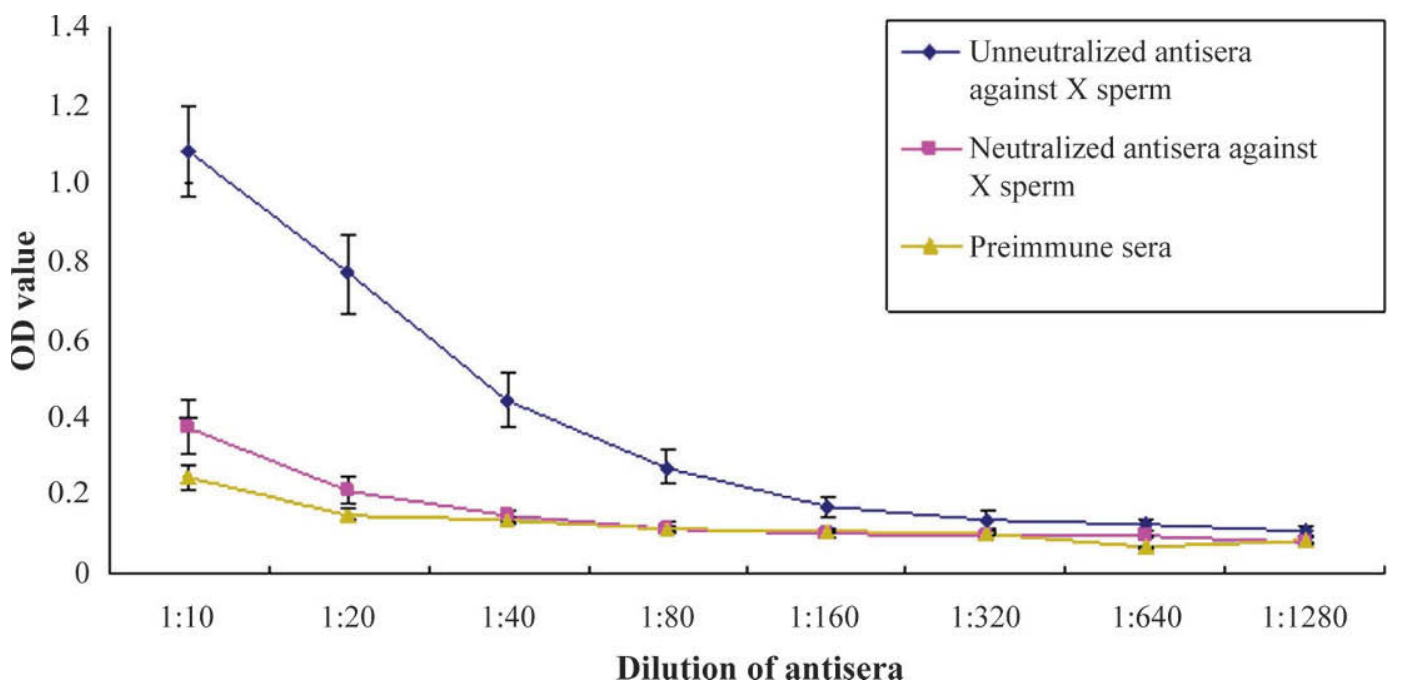

Figure 2. Neutralization test of different dilutions of antisera against sex-sorted X-sperm with excess unsorted sperm. The rabbit antisera against sex-sorted X-sperm were serially diluted ranging from 1:10 to 1:1,280 in PBS and incubated with an equal volume of $1 \times 10^{8} / \mathrm{mL}$ unsorted sperm, respectively. Following centrifugation treatment, the supernatants were collected and then evaluated for antibodies with a cellbased ELISA using unsorted sperm as antigen; the resulting optical density (OD) was measured at $450 \mathrm{~nm}$ in a plate reader (Multiskan MK3, Thermo Labsystems, Franklin, MA). When rabbit antisera against sex-sorted X-sperm were diluted $>1: 40$, no significant difference in mean OD values between the preimmune sera and the neutralized rabbit antisera against sex-sorted X-sperm was found $(P>0.05)$, and almost all of the antibodies against sex-sorted X-sperm were neutralized. The results are shown as mean values, and the error bars represent the standard deviations. Color version available in the online PDF.

\section{DISCUSSION}

The identification of a sex-specific plasma membrane protein in X- or Y-sperm would be useful to develop an immunological technique for separating sperm. How- ever, many attempts to identify a sex-specific protein have failed (Hendriksen et al., 1996; Howes et al., 1997; Hendriksen, 1999). Much indirect evidence has implied the existence of the SSP (Grant et al., 2008; Robbins et al., 2008; Zhang et al., 2008). As antibodies have specific

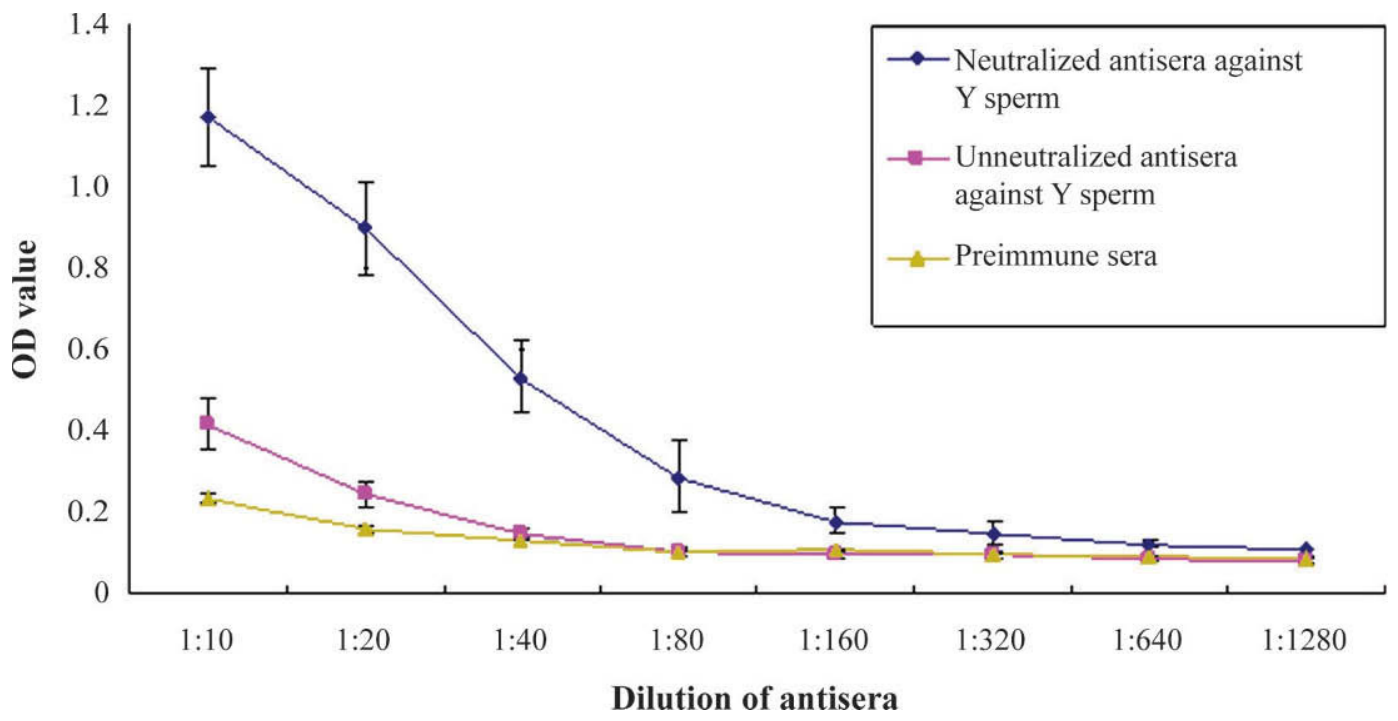

Figure 3. Neutralization test of different dilutions of antisera against sex-sorted Y-sperm with excess unsorted sperm. The rabbit antisera against sex-sorted Y-sperm were serially diluted ranging from 1:10 to 1:1,280 in PBS and incubated with an equal volume of $1 \times 10^{8} / \mathrm{mL}$ unsorted sperm, respectively. Following centrifugation treatment, the supernatants were collected and then evaluated for antibodies with a cellbased ELISA using unsorted sperm as antigen; the resulting optical density (OD) was measured at $450 \mathrm{~nm}$ in a plate reader (Multiskan MK3, Thermo Labsystems, Franklin, MA). When rabbit antisera against sex-sorted Y-sperm were diluted $\geq 1: 40$, no significant difference in mean OD values between the preimmune sera and the neutralized rabbit antisera against sex-sorted Y-sperm was found $(P>0.05)$, and almost all of the antibodies against sex-sorted Y-sperm were neutralized. The results are shown as mean values, and the error bars represent the standard deviations. Color version available in the online PDF. 

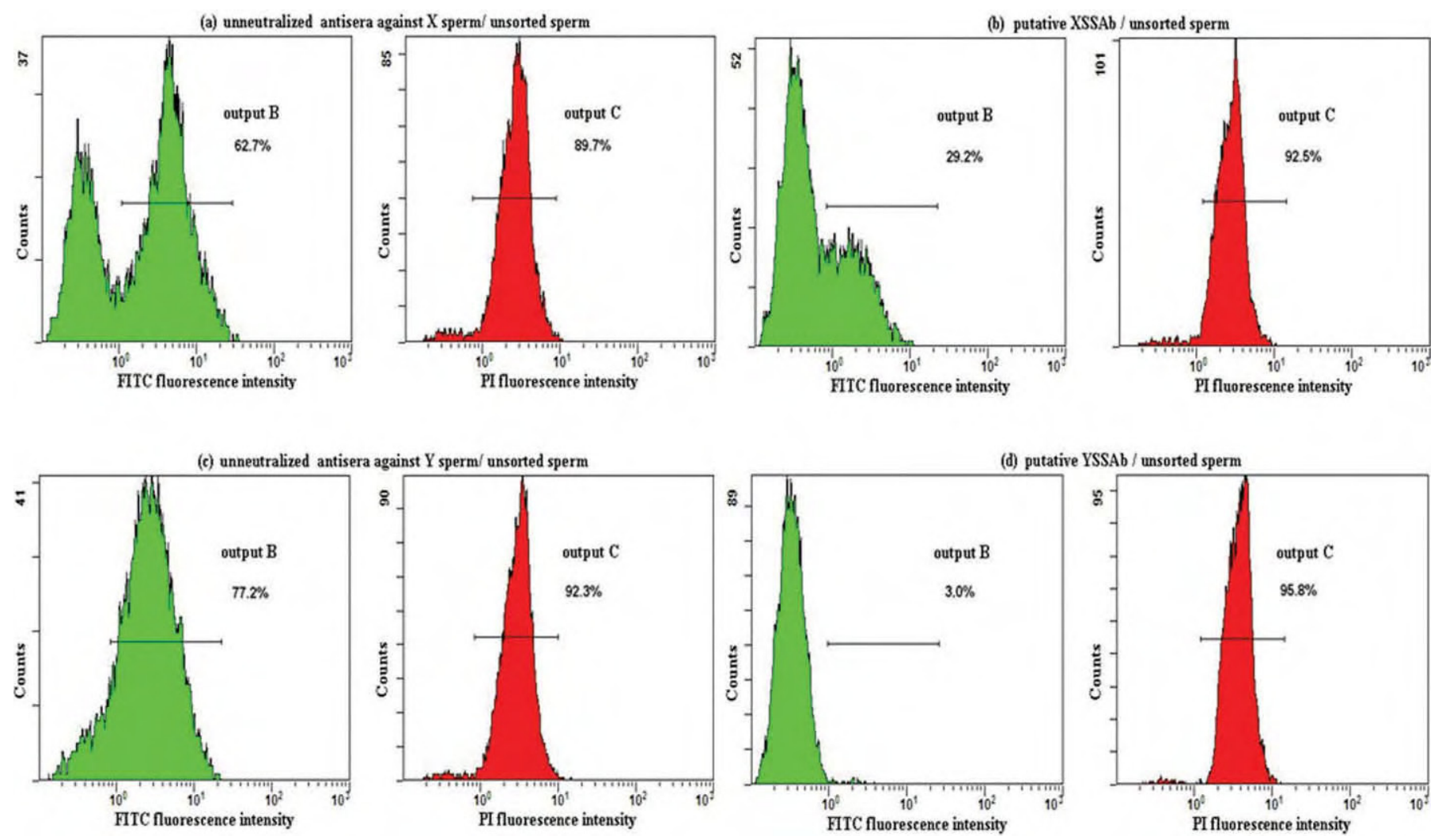

Figure 4. Screening of sex-specific antibodies (SSAb) by flow cytometry using unsorted sperm. (a) The unsorted sperm were incubated with the unneutralized rabbit antisera against X-sperm, (b) the putative SSAb against X-sperm (XSSAb), (c) the unneutralized rabbit antisera against Y-sperm, and (d) putative SSAb against Y-sperm (YSSAb). Following staining with fluorescein isothiocyanate (FITC)-conjugated goat anti-rabbit polyclonal antibodies and propidium iodide (PI), sperm were subjected to flow cytometric analysis. The flow cytometric histogram output $\mathrm{C}$ shows that mostly sperm were recognized by PI staining to exclude degenerated fragment of sperm and the other particles (PI fluorescence is reported on the $\mathrm{x}$-axis and counts on the $\mathrm{y}$-axis). Simultaneously, the recognized sperm were displayed in the histogram output B, and it shows that the sperm were bound by antibodies in the antisera as described above with immunofluorescence staining (FITC fluorescence is reported on the $\mathrm{x}$-axis and counts on the $\mathrm{y}$-axis). Color version available in the online PDF.

ability to recognize each cellular antigen, the immunological methods would provide great opportunities for identification of the SSP, and they have been used to identify sperm binding to zona pellucida (Sivapurapu et al., 2003), and to isolate capacitation-related antigens (Mollova et al., 1999) and other antigens with interesting functions (Khan et al., 2009). In this study, rabbits immunized with intact $\mathrm{X}$ - or $\mathrm{Y}$-sperm were used to produce SSAb based on the ability of the antibodies to recognize each cellular protein, respectively. However, detection of SSAb was severely hampered by the crossreactive antibodies against X- or Y-sperm. An immunoneutralization procedure could be used to decrease the interferences of cross-reactive antibodies. Wang et al. (2003) employed cross-reactive bacteria to neutralize serum prepared from rabbits immunized with vibrio, for

Table 1. The screening of antibodies in either neutralized rabbit antisera against sorted sperm (sex-specific antibodies against X- or Y-sperm) or unneutralized rabbit antisera against sorted sperm binding to unsorted bovine sperm

\begin{tabular}{lccc}
\hline & \multicolumn{2}{c}{ Treatment } \\
\cline { 2 - 3 } Type of antisera & 29.2 & Non-neutralization (\%) & Proportion $^{1}(\%)$ \\
\hline Antisera against X-sperm & 3.0 & 62.7 & 46.6 \\
Antisera against Y-sperm & & 77.2 & 3.9 \\
\hline
\end{tabular}

${ }^{1}$ The proportion of the sperm labeled by the neutralized antisera to the sperm labeled by the unneutralized antisera.

${ }^{2}$ The neutralization reaction of antisera against sorted sperm with excess sorted opposite-sex sperm. 

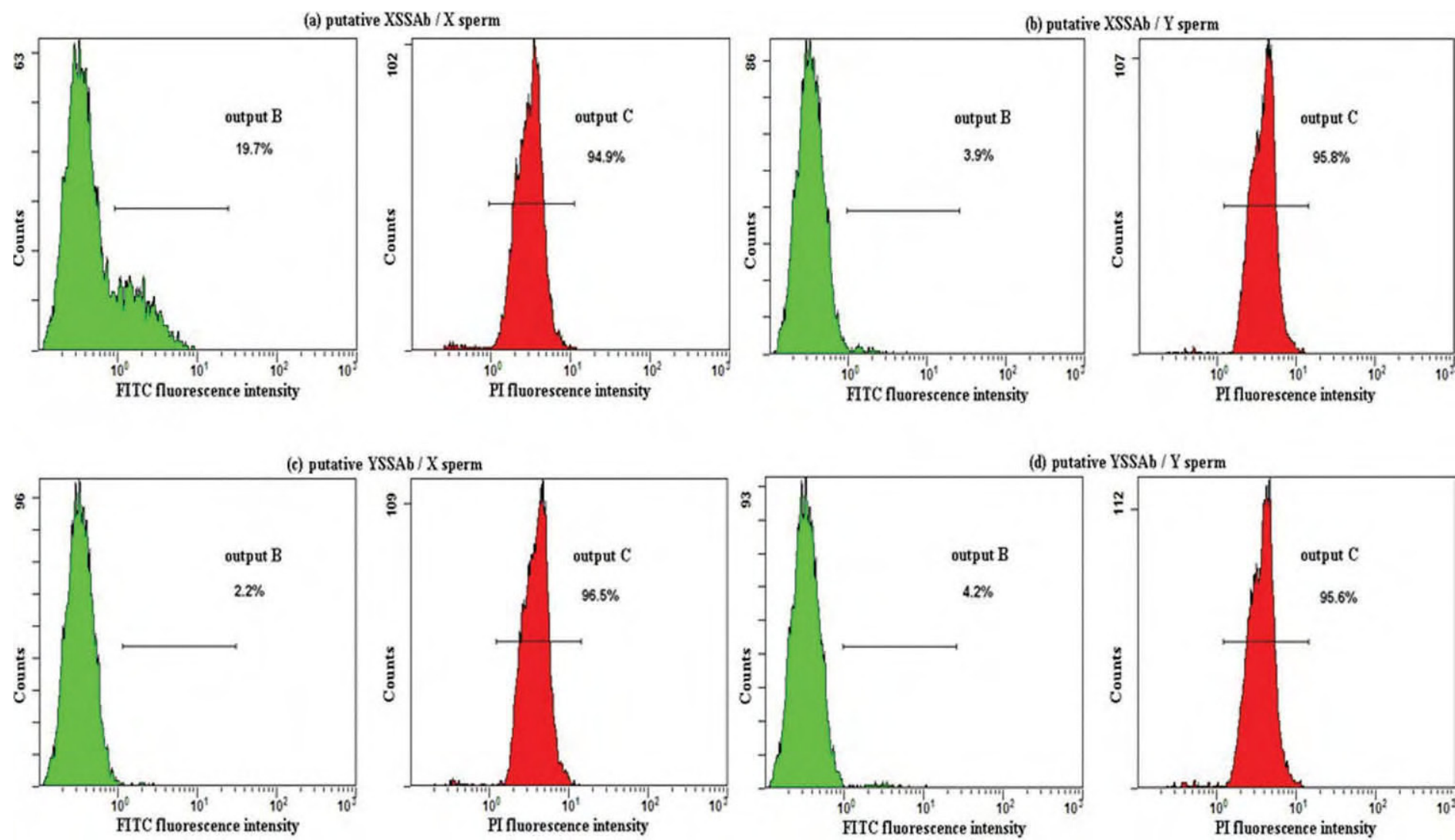

Figure 5. Screening of sex-specific antibodies (SSAb) by flow cytometry with sex-sorted X-and Y-sperm. The putative SSAb against $\mathrm{X}$-sperm (XSSAb) were incubated with either X-sperm (a) or Y-sperm (b), and simultaneously, the putative SSAb against Y-sperm (YSSAb) were incubated with either X-sperm (c) or Y-sperm (d). Following staining with fluorescein isothiocyanate (FITC)-conjugated goat anti-rabbit polyclonal antibodies and propidium iodide (PI), sperm were subjected to flow cytometric analysis. The flow cytometric histogram output $\mathrm{C}$ shows that most sperm were recognized by PI staining to exclude fragments of degenerated sperm and the other particles (PI fluorescence is reported on the $\mathrm{x}$-axis and counts on the $\mathrm{y}$-axis). Simultaneously, the recognized sperm were displayed in the histogram output B, and it shows that the sperm were bound by antibodies in the antisera as described above with immunofluorescence staining (FITC fluorescence is reported on the $\mathrm{x}$-axis and counts on the $\mathrm{y}$-axis). Color version available in the online PDF.

purification of specific antibodies against vibrio. Amital et al. (2007) removed most of the anti-VRT101 antibodies in lupus patients' sera using SyntheticVRT101. Hoffart et al. (2007) adopted erythrocyte-ghosts carrying antigens encapsulated inside polymeric and porous microparticles to decrease alloantibodies in blood significantly. Thus, the immuno-neutralization method provides an alternative technology to remove interference of cross-reactive antibodies, In this study, excess sex-sorted Y- or X-sperm were used to neutralize the cross-reactive antibodies in the rabbit antisera against $\mathrm{X}$ - or Y-sperm on the hypothesis that bovine proteins may be generally classified into types: non-sex-specific antibodies and sex-specific antigens (Ohno, 1967), and most of the non-sex-specific antibodies were decreased.

The SSAb in purified rabbit antisera against X- or Ysperm were detected on unsorted sperm and sex-sorted sperm by indirect fluorescence staining, in conjunction with flow cytometry analysis. The putative XSSAb or
YSSAb was initially selected by unsorted bovine sperm, respectively. The results showed that the putative XSS$\mathrm{Ab}$ bound $29.2 \%$ of unsorted sperm, and the putative YSSAb bound $3.0 \%$ of unsorted sperm, and it seemed that no SSAb were present in the putative XSSAb or YSSAb. However, as a consequence of the integrity at the sperm surface (Rubio-Guillén et al., 2006; Sancho et al., 2007), SSAb might be lost with the criterion that the antibodies bound to 40 to $60 \%$ of unsorted sperm cells without positive control. In this study, the unneutralized antisera against sex-sorted sperm were used as a positive control to avoid losing SSAb, and the sperm labeled by the putative XSSAb accounted for $46.6 \%$ of unsorted sperm recognized by the non-neutralized antisera against X-sperm (29.2\%: 62.7\%). However, an antibody which consistently binds to about $40 \%$ to $60 \%$ of the unsorted sperm population could not be simply recognized as SSAb (Soares and Barbosa, 2008), and might be associated with surface antigens that are not 
1

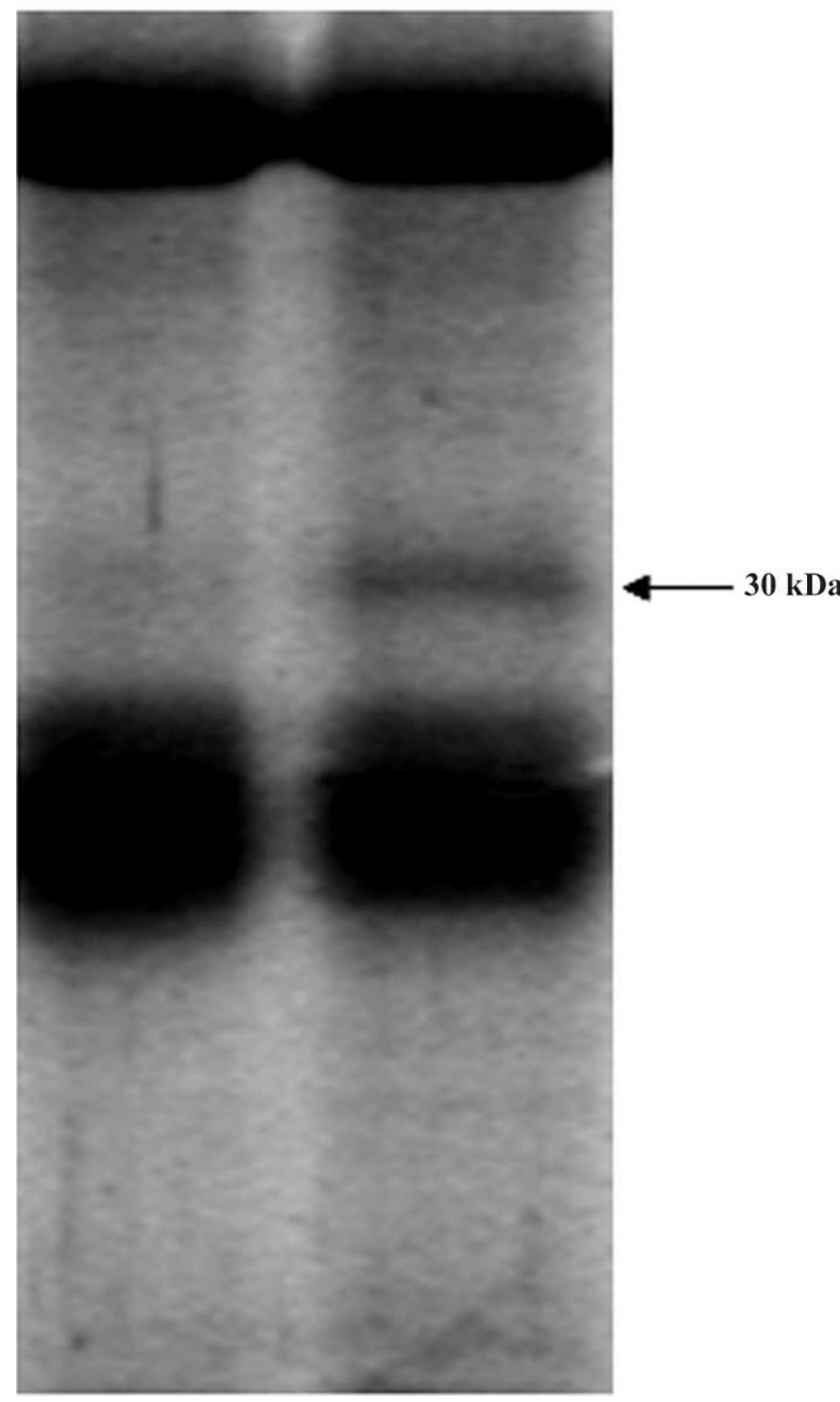

Figure 6. Capture of sex-specific proteins (SSP) in unsorted sperm protein by the putative sex-specific antibodies against X-sperm (XSSAb) and sex-specific antibodies against Y-sperm (YSSAb) using immunoprecipitation. The SSP in unsorted sperm protein were captured by the putative XSSAb or YSSAb using immunoprecipitation Then, the immunocomplexes were subjected to SDS-PAGE and gels were silver stained: 1st lane: the proteins captured by the putative YSSAb, 2nd lane: the proteins captured by the putative XSSAb. The arrow shows a specific band of approximately $30 \mathrm{kDa}$ in lane 2 , but not present in detectable levels in lane 1.

present on all sperm but whose distribution is unrelated to the presence of either sex chromosome (Howes et al., 1997). Thus, it was necessary to be further validated by X-and Y-sperm, respectively. In regard to this, the putative XSSAb or YSSAb were submitted to binding assay against $\mathrm{X}$ - and $\mathrm{Y}$-sperm by flow cytometry, respectively, and only the putative XSSAb, which bound $19.7 \%$ of X-sperm and $3.9 \%$ of Y-sperm, showed specificity to X-sperm. These results implied that SSAb might be present in the putative XSSAb.

For further verification of the SSAb and capture of SSP, the putative XSSAb or YSSAb were used to capture SSP from a broad panel of solubilized sperm proteins, respectively, and a sex-specific protein (about $30 \mathrm{KDa}$ ) was specifically captured by the putative XSSAb. A similar result was obtained by Howes et al. (1997) using 2-dimensional electrophoresis to distinguish differences between the whole proteins of X- and Y-sperm, but the sex-specific protein was not detected in membrane proteins. These results were explained as follows: the extraction of sperm plasma membrane proteins failed to extract bovine sperm thoroughly, leaving SSP in sperm residue, or SSP might be mostly lost in the extraction of sperm membrane proteins and were below the detection level (Howes et al., 1997; Wang et al., 2006; Bermejo-Álvarez et al., 2008). Therefore, the whole sperm proteins were resolved in denaturing lysis buffer to extract sperm thoroughly, and SSP could be further concentrated by immunoprecipitation at the same with captured by SSAb (Harlow and Lane, 1999). Meanwhile, the results of screening of SSAb by flow cytometer indicated that the putative XSSAb sperm showed differential binding to $\mathrm{X}$ and $\mathrm{Y}$ chromosomebearing sperm, respectively; Blecher et al. (1999) also reported that the female rabbit sera against unsorted sperm could cause agglutination of approximately half of the bovine sperm; when the unagglutinated sperm were isolated and used in bovine in vitro fertilization, they produced above $90 \%$ male embryos. Thus, we hypothesized that our antibodies capture a specific protein present in subpopulations of sex-sorted sperm.

The protein composition of X- and Y-sperm can only be different when $\mathrm{X}$ - and Y-chromosomal genes are expressed after meiosis and when the gene products are not to be transported to other spermatids by means of the intercellular bridges (Hendriksen, 1999). Increasing evidence for post-meiotic transcription of genes on both sex-chromosomes exists. Examples of genes on the X chromosome expressed in spermatids are Span (Westbrook et al., 2006), Ssxb (Mueller et al., 2008), Akap 4 (Stouffs et al., 2009), Magec2 (Güre et al., 2000); Zfy (Hansen et al., 2006), Sly (Cocquet et al., 2009), and Ssty1 (Turner and Burgoyne, 2007). However, studies have provided evidence for sharing of gene products among spermatids by way of cytoplasmic bridges that link post-meiotic spermatids (Riparbelli and Callaini, 2007; Immler, 2008; Zuccarello et al., 2009). On the other hand, it was proven that gene products were not all exchange via the intercellular cytoplasmic bridges (Hendriksen, 1999). In spite of this, the direct evidence for certain gene products to be more abundantly pres- 
ent in half of the spermatids has not been provided, although a few examples implied differences in protein composition between X- and Y-sperm (Grant et al., 2008; Robbins et al., 2008; Zhang et al., 2008). In this study, the presence of a sex-specific protein (about 30 $\mathrm{kDa}$ ) in bovine $\mathrm{X}$-sperm might provide further evidence that the exchange among sister spermatids via the intercellular cytoplasmic bridges does not occur for all gene products.

\section{CONCLUSIONS}

In summary, a series of immunologic methods was adopted to screen and identify SSAb in the putative XSSAb or YSSAb, and the results showed that SSAb were confirmed in the putative XSSAb; a sex-specific protein (about $30 \mathrm{kDa}$ ) also was captured by the antisera. Sex-specific proteins might be present on the Xsperm surfaces and may offer some assistance to exploit an immunological procedure for sexing sperm. Further studies are required to confirm the uniqueness of the protein and its potential applications.

\section{ACKNOWLEDGMENTS}

The authors wish to thank Breeding Bull Station of Hubei Province (Wuhan, China) for providing us with semen from Holstein bulls. This study is supported by Program 863 (2008AA101010), Modern Agriculture Technology Research System (nycytx-10) and Government Financial Support Project of China (No.2006BAD04A02-11).

\section{REFERENCES}

Amital, H., M. Heilweil-Harel, R. Ulmansky, M. Harlev, E. Toubi, A. Hershko, and Y. Naparstek. 2007. Antibodies against the VRT101 laminin epitope correlate with human SLE disease activity and can be removed by extracorporeal immunoadsorption. Rheumatology 46:1433-1437.

Beerli, R. R., M. Bauer, R. B. Buser, M. Gwerder, S. Muntwiler, P. Maurer, P. Saudan, and M. F. Bachmann. 2008. Isolation of human monoclonal antibodies by mammalian cell display. Proc. Natl. Acad. Sci. USA 105:14336-14341.

Bermejo-Álvarez, P., D. Rizos, D. Rath, P. Lonergan, and A. Gutiérrez-Adán. 2008. Can bovine in vitro-matured oocytes selectively process X- or Y-sorted sperm differentially? Biol. Reprod. 79:594-597.

Blecher, S. R., R. Howie, S. Li, J. Detmar, and L. M. Blahut. 1999. A new approach to immunological sexing of sperm. Theriogenology 52:1309-1321.

Cocquet, J., P. J. I. Ellis, Y. Yamauchi, S. K. Mahadevaiah, N. A. Affara, M. A. Ward, and P. S. Burgoyne. 2009. The multicopy gene Sly represses the sex chromosomes in the male mouse germline after meiosis. PLoS Biol. 7:e1000244.doi:10.1371/journal. pbio. 1000244 .

Garner, D. L., and G. E. Seidel. 2008. History of commercializing sexed semen for cattle. Theriogenology 69:886-895.

Grant, V. J., R. J. Irwin, N. T. Standley, A. N. Shelling, and L. W. Chamley. 2008. Sex of bovine embryos may be related to mothers' preovulatory follicular testosterone. Biol. Reprod. 78:812-815.
Güre, A. O., E. Stockert, K. C. Arden, A. D. Boyer, C. S. Viars, M. J. Scanlan, L. J. Old, and Y.-T. Chen. 2000. CT10: A new cancer-testis (CT) antigen homologous to CT7 the MAGE family identified by representational-difference analysis. Int. J. Cancer 85:726-732.

Hansen, M. A., J. E. Nielsen, M. Tanaka, K. Almstrup, N. E. Skakkebaek, and H. Leffers. 2006. Identification and expression profiling of 10 novel spermatid expressed CYPT genes. Mol. Reprod. Dev. $73: 568-579$

Harlow, E., and D. Lane. 1999. Using Antibodies: A Laboratory Manual. Cold Spring Harbor Laboratory Press, New York, NY.

Hendriksen, P. J. 1999. Do X and Y spermatozoa differ in proteins? Theriogenology 52:1295-1307.

Hendriksen, P. J., G. R. Welch, J. A. Grootegoed, T. Van der Lende, and L. A. Johnson. 1996. Comparison of detergent-solubilized membrane and soluble proteins from flow cytometrically sorted $\mathrm{X}$ - and Y-chromosome bearing porcine spermatozoa by high resolution 2-D electrophoresis. Mol. Reprod. Dev. 45:342-350.

Hoffart, V., P. Maincent, A. Lamprecht, V. Latger-Cannard, V. Regnault, C. Merle, V. Jouan-Hureaux, T. Lecompte, C. Vigneron, and N. Ubrich. 2007. Immunoadsorption of alloantibodies onto erythroid membrane antigens encapsulated into polymeric microparticles. Pharm. Res. 24:2055-2062.

Howes, E. A., N. G. Miller, C. Dolby, A. Hutchings, G. W. Butcher, and R. Jones. 1997. A search for sex-specific antigens on bovine spermatozoa using immunological and biochemical techniques to compare the protein profiles of $\mathrm{X}$ and $\mathrm{Y}$ chromosome-bearing sperm populations separated by fluorescence-activated cell sorting. J. Reprod. Fertil. 110:195-204.

Immler, S. 2008. Sperm competition and sperm cooperation: The potential role of diploid and haploid expression. Reproduction 135:275-283.

Karabinus, D. S. 2009. Flow cytometric sorting of human sperm: MicroSort clinical trial update. Theriogenology 71:74-79.

Khan, S. A., A. R. Suryawanshi, S. A. Ranpura, S. V. Jadhav, and V. V. Khole. 2009. Identification of novel immunodominant epididymal sperm proteins using combinatorial approach. Reproduction 138:81-93.

Liang, A., S. Cao, L. Han, Y. Yao, M. Moaeen-ud-Din, and L. Yang. 2008. Construction and evaluation of the eukaryotic expression plasmid encoding two copies of somatostatin genes fused with hepatitis B surface antigen gene S. Vaccine 26:2935-2941.

Mollova, M., T. Djarkova, M. Ivanova, M. Stamenova, and S. Kyurkchiev. 1999. Isolation and biological characterization of boar sperm capacitation-related antigen. Am. J. Reprod. Immunol. 42:254-262.

Mueller, J. L., S. K. Mahadevaiah, P. J. Park, P. E. Warburton, D. C. Page, and J. M. A. Turner. 2008. The mouse X chromosome is enriched for multicopy testis genes showing postmeiotic expression. Nat. Genet. 40:794-799.

O'Brien, J. K., K. J. Steinman, and T. R. Robeck. 2009. Application of sperm sorting and associated reproductive technology for wildlife management and conservation. Theriogenology 71:98-107.

Ohno, S. 1967. Sex Chromosomes and Sex-Linked Genes. SpringerVerlag, Berlin, Germany.

Rajeev, S. K., and K. V. R. Reddy. 2004. Sperm membrane protein profiles of fertile and infertile men: Identification and characterization of fertility-associated sperm antigen. Hum. Reprod. 19:234242.

Rath, D., G. Moench-Tegeder, U. Taylor, and L. A. Johnson. 2009. Improved quality of sex-sorted sperm: A prerequisite for wider commercial application. Theriogenology 71:22-29.

Riparbelli, M. G., and G. Callaini. 2007. The Drosophila parkin homologue is required for normal mitochondrial dynamics during spermiogenesis. Dev. Biol. 303:108-120.

Robbins, W. A., F. S. Wei, D. A. Elashoff, G. P. Wu, L. Xun, and J. Jia. 2008. Y:X sperm ratio in boron-exposed men. J. Androl. 29:115-121.

Rubio-Guillén, J., D. González, J. J. Garde, M. C. Esteso, M. R. Fernández-Santos, J. E. Rodríguez-Gíl, N. Madrid-Bury, and A. Quintero-Moreno. 2006. Effects of cryopreservation on bull sper- 
matozoa distribution in morphometrically distinct subpopulations. Reprod. Domest. Anim. 42:354-357.

Sambrook, J., and D. W. Russell. 2001. Molecular Cloning: A Laboratory Manual. 3rd ed. Cold Spring Harbor Laboratory Press, New York, NY.

Sancho, S., I. Casas, H. Ekwall, F. Saravia, H. Rodriguez-Martinez, J. E. Rodriguez-Gil, E. Flores, E. Pinart, M. Briz, N. Garcia-Gil, J. Bassols, A. Pruneda, E. Bussalleu, M. Yeste, and S. Bonet. 2007. Effects of cryopreservation on semen quality and the expression of sperm membrane hexose transporters in the spermatozoa of Iberian pigs. Reproduction 134:111-121.

Seidel, G. E. 2009. Sperm sexing technology - The transition to commercial application. An introduction to the symposium "Update on sexing mammalian sperm". Theriogenology 71:1-3.

Sivapurapu, N., A. Upadhyay, A. Hasegawa, K. Koyama, and S. K. Gupta. 2003. Efficacy of antibodies against Escherichia coli expressed chimeric recombinant protein encompassing multiple epitopes of zona pellucida glycoproteins to inhibit in vitro human sperm-egg binding. Mol. Reprod. Dev. 65:309-317.

Soares, S. G., and J. E. Barbosa. 2008. Application of recombinant antibody library for screening specific antigens in a bovine sperm cell subpopulation. Livest. Sci. 114:188-193.

Spinaci, M., S. Volpe, C. Bernardini, M. de Ambrogi, C. Tamanini, E. Seren, and G. Galeati. 2006. Sperm sorting procedure induces a redistribution of Hsp70 but not Hsp60 and Hsp90 in boar spermatozoa. J. Androl. 27:899-907.

Stouffs, K., H. Tournaye, I. Liebaers, and W. Lissens. 2009. Male infertility and the involvement of the X chromosome. Hum. Reprod. Update 15:623-637.
Turner, J. M. A. and P. S. Burgoyne. 2007. Male meiotic sex chromosome inactivation and meiotic silencing. Pages $27-45$ in The Y Chromosome and Male Germ Cell Biology in Health and Diseases. Y.-F. C. Lau and W.-Y. Chan, ed. World Scientific Publishing Co., Singapore.

Wang, J., Y. Su, and Q. Yan. 2003. A fast and indirect fluorescent antibody assay for the vibrio in large yellow croaker Pseudosciaena crocea (Richardson). Chin. J. Oceanology Limnol. 21:91-96.

Wang, Z. L., D. Wang, H. B. Zhu, J. H. Cheng, Y. B. Li, H. S. Hao, W H. Du, and L. J. Sha. 2006. The study on separating the protein of bovine sperm by two-dimensional gel electrophoresis. Chin. J. Biotechnol. 26:61-66.

Westbrook, V. A., P. D. Schoppee, G. R. Vanage, K. L. Klotz, A B. Diekman, C. J. Flickinger, M. A. Coppola, and J. C. Herr. 2006. Hominoid-specific SPANXA/D genes demonstrate differential expression in individuals and protein localization to a distinct nuclear envelope domain during spermatid morphogenesis. Mol. Hum. Reprod. 12:703-716.

Zhang, L., W. Du, X. Lin, A. Zhang, and H. Chen. 2008. Progesterone and 17 beta-estradiol, but not follicle stimulating hormone, alter the sex ratio of murine embryos fertilized in vitro. Theriogenology 69:961-966.

Zuccarello, D., A. Garolla, A. Ferlin, M. Menegazzo, L. De Toni, M. Carraro, C. Veronese, and C. Foresta. 2009. Androgen receptor is expressed in both X- and Y-carrier human spermatozoa. Fertil. Steril. 91:193-200. 